\title{
Interaction of reward magnitude and conditioned fear on the consummatory response'
}

JOHN R. VOGEL AND NORMAN E. SPEAR

RUTGERS UNIVERSITY

Three experiments are reported in which tone offset was associated with shock using a modified CER procedure. Ss were then tested for response suppression to the tone when given $4,11.5$ or $32 \%$ sucrose solution. Results indicated that there was less response suppression in the $32 \%$ Ss compared to the $4 \%$ Ss. This persisted despite wide variations in values of critical parameters.

The present experiments emerged from an attempt to find a simple procedure sensitive to variations in reinforcer magnitude. Although this variable reliably affects behavior in simple tasks, such as the straight runway, the considerable overlap in response distributions and the small absolute differences often obtained as a function of reinforcer magnitude are well known. This report describes three variations of a method useful to such an investigation. Its advantages are that: (a) No shaping of behavior is required; the response (licking) is immediately available in S's repertoire, (b) the training procedure is short, simple, and requires relatively little tampering with $S$ 's behavioral history, and (c) the effects of reinforcer magnitude are quite robust.

The basic procedure was a modification of the EstesSkinner CER technique further developed by Leaf \& Muller (1965). This consists of measuring the extent to which the presentation of a stimulus $\left(\mathrm{S}^{\mathrm{F}}\right)$, previously associated with shock, leads to a disruption of ongoing consummatory behavior. Geller (1960) used the conventional CER procedure, and its required extensive training, to compare suppression of a lever-press response with different base line reinforcers. He reported that Ss rewarded with a solution of sweetened condensed milk were more resistant to suppression than Ss responding for water. The present experiments investigated response suppression using systematic variations within a single reinforcer dimension-sweetness of a sucrose solution:

\section{Method}

\section{EXPERIMENT I}

The Ss were 10 adult male, experimentally naive, Sprague-Dawley rats. The apparatus was a modified Gerbrands Student Demonstration Box (levers removed and water bottle with glass drinking tube fitted to one wall of the box and a 4-in. speaker mounted near the drinking tube). A dim light illuminated the box, and the entire apparatus was set in a large sound-attenuated enclosure. A ventilating fan helped to mask sounds from the programming equipment. A drinkometer was connected to the drinking tube and the floor so that S com- pleted the circuit whenever he licked the tube. A GrasonStadler shocker set at $1 \mathrm{ma}$ for $1 \mathrm{sec}$. was used during training.

The Ss were placed on a deprivation schedule of $10 \mathrm{gm}$ powdered Purina Chow per day for 21 days prior to experimental training. Then on each of five days, $S$ was allowed to make 110 licks at the drinking tube which contained either $4 \%$ (Group $4-5 \mathrm{Ss}$ ) or $32 \%$ (Group 32$5 \mathrm{Ss})$ sucrose solution. On Day 6 , the drinking tube was removed. The $\mathrm{S}$ was placed in the apparatus, and after $60 \mathrm{sec} .$, a tone of about $1000 \mathrm{cps}$ at moderate intensity was turned on for $10 \mathrm{sec}$. Termination of the tone was accompanied by a shock of $1 \mathrm{ma}$ for $1 \mathrm{sec}$. On Day 7, the tube was replaced and filled with the appropriate solution. Following S's completion of 100 licks, the tone came on. The time to complete 10 licks in the presence of the tone was recorded.

Results

A two-tailed Mann-Whitney U test showed that Group 4 took significantly longer $(U=1, p<.016)$ to complete 10 licks during the tone than Group 32 (see Fig. 1). In fact, four of the five Ss in Group 4 did not complete 10 licks in the $5 \mathrm{~min}$. test period. Another comparison is provided by examining time to complete 10 licks after the first 100 licks of Day 5 (before tone-shock pairing; no tone presented) with that of Day 7 (after tone-shock pairing; with tone presented). A comparison of these differences for each $S$ indicated that the amount of suppression

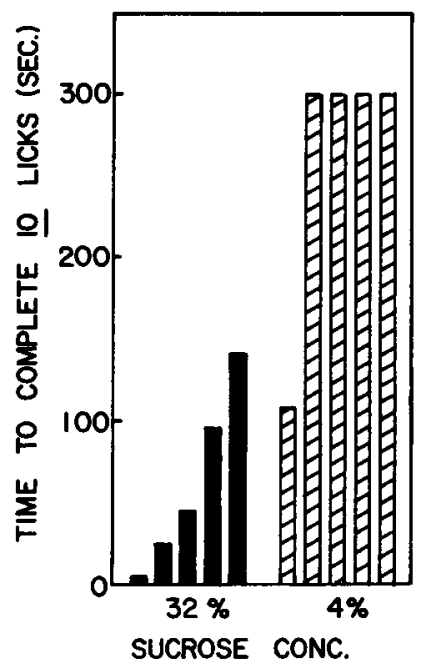

Fig. 1. Individual times to complete 10 licks at a drinking tube containing the indicated solution while in the presence of a tone previously associated with shock in Experiment 1. 
produced by $\mathrm{S}^{\mathrm{F}}$ was reliably greater in Group 4 than in Group $32(U=1, p<.016)$. In fact, the rank order of Ss was not changed by this measure.

\section{Method}

\section{EXPERIMENT II}

The Ss were 12 adult male Sprague-Dawley rats. These animals previously served as Ss in a maze study which incorporated food deprivation and food pellet incentives. The apparatus was that used in Experiment $I$, and the procedure was similar except that Ss were given only two days of pretraining with the assigned sucrose concentration. Also, two, rather than one, tone-shock pairings were given on Day 3 with an intertrial interval of $60 \mathrm{sec}$. An apparatus failure resulted in one $S$ in Group 32 receiving two tone-shock pairings of 2 ma shock for 2 sec.

\section{Resulis}

Again Group 4 took significantly longer $(U=2, p<.008)$ to complete 10 licks in the presence of the tone than did Group 32 (see Fig. 2). The increased shock appeared to have little effect (see $S$ in Group 32 marked with an arrow). A comparison of scores on Day 2 with those on Day 4 indicated that $\mathrm{S}^{\mathrm{F}}$ produced more suppression in Group 4 than in Group $32(\mathrm{U}=3, \mathrm{p}<.016)$.

It was expected that increased tone-shock training would lead to greater suppression and, therefore, Ss in Experiment II would show greater suppression than those in Experiment I. Groups 4 did not differ. However, since most of these Ss reached the 5 min.criterion, a ceiling effect may have masked any differences. It was surprising that Groups 32 did not differ between Experiment I and II; and this cannot be attributed to a ceiling effect. Perhaps the simple additional tone-shock pairing was not sufficient to produce a measurable increase in the fear-

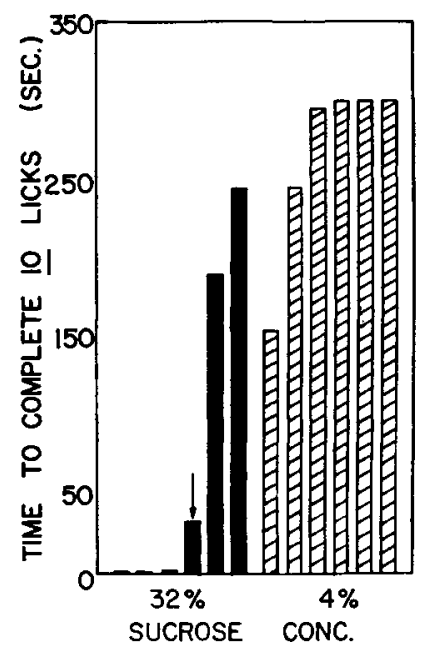

Fig. 2. Individual times to complete 10 licks in the presence of the tone in Experiment 2. producing properties of $\mathrm{S} F$. Despite differences in the procedures of Experiments I and II, equally large differences occurred between the 4 and $32 \%$ groups.

A third experiment incorporated another change in procedure and added a third group, trained and tested on $11.5 \%$ solution.

\section{Method}

\section{EXPERIMENT III}

Fifteen adult male, Sprague-Dawley rats were used which had previously served as Ss in a maze study incorporating food deprivation and food pellet incentives. They were placed on food and water deprivation prior to training. On Day 1, $\mathrm{S}$ was allowed to make 110 licks at the drinking tube which contained either 4\% (Group 4$5 \mathrm{Ss}$ ), $11.5 \%$ (Group $11.5-5 \mathrm{Ss}$ ), or $32 \%$ (Group $32-5 \mathrm{Ss}$ ) sucrose solution. At the end of this session, all Ss were put on ad lib water. On Day 2, $\mathrm{S}$ was placed in the apparatus with the drinking tube removed. After $30 \mathrm{sec}$. a tone of about 1000 cps and moderate intensity was turned on for $5 \mathrm{sec}$, and the termination of the tone was accompanied by a shock of $1 \mathrm{ma}$ for $1 \mathrm{sec}$. On Day 3, the tube was replaced and filled with the appropriate solution. Following $S^{\prime}$ 's completion of 100 licks, the tone came on. The time to complete 10 licks in the presence of the tone was recorded.

\section{Results and Discussion}

One-tailed Mann-Whitney $U$ tests indicated that Groups 4 and 11.5 took significantly longer $(U=2, p<.02$ for $4 \% ; \mathrm{U}=4, \mathrm{p}<.02$ for $11.5 \%$ ) to complete 10 licks during the tone than Group 32. Groups 4 and 11.5 did not differ. A comparison of suppression scores (see Experiments I and II) yielded similar results with no change in the rank order of Ss.

The failure to find a behavior difference between Ss given $11.5 \%$ and those given $4 \%$ sucrose solution may be attributed to the small amount of experience $\mathrm{S}$ had with its particular percentage sucrose and the shortened CS duration employed in Experiment III. However, the sizable behavior differences between Group 32 and that of Ss with lower percentages indicate that this procedure is robust in attaining behavioral differences as a function of reinforcer magnitude. The present three experiments show that these differences persist in spite of several variations in the value of critical parameters.

\section{References}

Geller, I. The acquisition and extinction of conditioned suppression as a function of the base-line reinforcer. J. exp. Anal. Behav., $1960,3,235-240$.

Leaf, R. C., \& Muller, S. A. Simple method for CER conditioning and measurement. Psychol. Rep., 1965, 17, 211-215.

\section{Note}

1. This research was supported by a grant-in-aid-of-research from the Sigma Xi foundation held by the first author and USPHS Grant MH-08888-02 held by the second author. 